\title{
SCIENTIFIC REPORTS

\section{Resistance training preserves high- intensity interval training induced improvements in skeletal muscle capillarization of healthy old men: a randomized controlled trial}

\begin{abstract}
Aurel B. Leuchtmann (1) 1,6,8, Sandro Manuel Mueller 2,3,4,8, David Aguayo ${ }^{3,7}$, Jens A. Petersen ${ }^{2}$, Maria Ligon-Auer ${ }^{3}$, Martin Flück ${ }^{5}$, Hans H. Jung ${ }^{2}$ \& Marco Toigo ${ }^{3,4,5^{*}}$

Skeletal muscle capillarization is a determining factor in gas and metabolite exchange, while its impairments may contribute to the development of sarcopenia. Studies on the potential of resistance training (RT) to induce angiogenesis in older muscles have been inconclusive, and effects of sequential endurance training (ET) and RT on capillarization are unknown. Healthy older men ( $66.5 \pm 3.8$ years) were engaged in either 12 weeks of habitual course observation (HC) followed by 12 weeks of $R T(n=8)$, or 12 weeks of high-intensity interval training (HIIT) followed by 12 weeks of RT $(n=9)$. At baseline, following 12 and 24 weeks, $m$. vastus lateralis biopsies were obtained. (Immuno-)histochemistry was used to assess indices of muscle fiber capillarization, muscle fiber morphology and succinate dehydrogenase (SDH) activity. Single periods of RT and HIIT resulted in similar improvements in capillarization and SDH activity. During RT following HIIT, improved capillarization and SDH activity, as well as muscle fiber morphology remained unchanged. The applied RT and HIIT protocols were thus similarly effective in enhancing capillarization and oxidative enzyme activity and RT effectively preserved HIIT-induced adaptations of these parameters. Hence, both, RT and HIIT, are valid training modalities for older men to improve skeletal muscle vascularization.
\end{abstract}

Aging is associated with reduced cardiorespiratory fitness ${ }^{1,2}$ and impaired responsiveness of skeletal muscle tissue to anabolic stimuli ${ }^{3-5}$. Skeletal muscle capillarization can affect both cardiorespiratory fitness and muscle anabolism. Enhanced capillarization ameliorates gas and metabolite exchange between blood and muscle tissue, resulting in improved peak oxygen uptake $\left(\dot{V} O_{2 p e a k}\right)$ and oxidative capacity ${ }^{6,7}$. Likewise, it has been proposed, that increased blood flow to muscles is required to support muscle fiber hypertrophy and satellite cell activation in older adults by facilitating the delivery of nutrients, cytokines and growth factors ${ }^{8,9}$. Furthermore, a recent study by Prior et al. ${ }^{10}$ revealed that sarcopenic older adults have lower values for capillarization indices when compared to non-sarcopenic controls. Taken together, emerging evidence suggests that a decline in capillarization with increasing age might contribute to the development of sarcopenia and functional impairments in older adults.

Endurance-type training (ET) is considered the method of choice for improving capillarization in skeletal muscle and inducing the associated local and systemic health-related benefits. While ET has consistently been demonstrated to stimulate angiogenesis and to substantially increase skeletal muscle capillarization and $\dot{V} O_{2 p e a k}$ in older adults $s^{6,711}$, data on the potential of resistance training (RT) to effectively induce angiogenesis in aged muscle has been inconclusive. Some studies reported an increase in capillarization following 9-24 weeks of

${ }^{1}$ Institute of Translational Medicine ITM, ETH Zurich, Zurich, Switzerland. ${ }^{2}$ Department of Neurology, University Hospital Zurich, Zurich, Switzerland. Institute of Human Movement Sciences, ETH Zurich, Zurich, Switzerland. ${ }^{4}$ Research and Performance Center for Elite Athleticism, OYM, Cham, Switzerland. ${ }^{5}$ Laboratory for Muscle Plasticity, Balgrist University Hospital, Department of Orthopaedics, University of Zurich, Zurich, Switzerland. ${ }^{6}$ Present address: Biozentrum, University of Basel, Klingelbergstrasse 50/70, $\mathrm{CH}-4056$, Basel, Switzerland. ${ }^{7}$ Present address: Kieser Training, Zurich, Switzerland. ${ }^{8}$ These authors contributed equally: Aurel B. Leuchtmann and Sandro Manuel Mueller. *email: marco.toigo@oym.ch 
$\mathrm{RT}^{6,9,11,12}$, but others observed no effect of RT on capillarization indices ${ }^{8,13,14}$. However, most of the studies investigating the effect of training on capillarization in older adults, considered either RT or ET groups, and did not include both training modalities. Consequently, it is not possible to infer from these studies whether there are adaptive differences of capillarization indices between the two training modalities.

To elucidate whether RT and ET differ in their effects on structural adaptations, the inclusion of both training modalities into one study together with a sequential training design is required. So far, only one study has investigated the effects of sequential RT and ET on capillarization ${ }^{6}$. The authors observed a similar increase in capillarization indices after 18 weeks of ET compared to 9 weeks of RT followed by 9 weeks of ET. More importantly, the increased capillary-to-fiber ratio and number of capillary contacts after 9 weeks of RT could not be further increased by the following 9 weeks of ET. These findings indicate that the two distinct and sequentially applied exercise modalities do not differ in their potential to increase capillarization indices in older adults. Furthermore, there appears to be a mechanistic overlap in the underlying adaptation processes for the studied population, as the subsequent ET did not act synergistically to further increase capillary supply. However, the reverse sequence, in which a period of ET precedes a period of RT, has not been investigated so far.

In this study, we aimed at investigating adaptation patterns of capillarization indices in study groups that were subjected to single periods of exclusive RT or ET in the form of high-intensity interval training (HIIT). We have chosen HIIT as the type of ET, because a solid body of evidence suggests that HIIT has similar if not superior effects on parameters quantifying ET adaptations, while being well tolerated even in diseased populations ${ }^{15}$. Importantly, compared to traditional endurance-based training, HIIT appears to be more time efficient and is generally perceived as less monotonous, which clearly helps to overcome issues with adherence and compliance. To further characterize possible adaptation differences between RT and ET, we imposed study groups to a period of RT without prior training or subsequent to a period of HIIT and compared capillarization indices.

\section{Methods}

Participants. Twenty older recreationally active men, recruited from the area of Zurich (Switzerland), voluntarily participated in this study. Participants were free of any musculoskeletal or other disorders that could potentially affect their ability to complete testing and/or training. They had no experience with systematic RT or ET for the previous 2 years prior to their enrolment. Two participants withdrew from the study for personal reasons not related to the study. An additional participant was excluded from the final analyses, because he did not fulfill the training frequency guidelines. In total, 17 participants $(66.5 \pm 3.8$ years, $82.4 \pm 13.0 \mathrm{~kg}, 177 \pm 5 \mathrm{~cm}$, $\left.2.28 \pm 0.37 \mathrm{~W} \cdot \mathrm{kg}^{-1}\right)$ completed the training intervention and all assessments. Prior to enrolment in the study, participants were fully informed about the purposes, benefits and risks associated with the study and completed a routine health questionnaire before giving written informed consent to their participation in the study. In case of a health-related doubt, consent of a physician was obtained. The study was approved by the ethics committee of the Canton of Zurich [Kantonale Ethik-Kommission Zürich (KEK) reference number: KEK-ZH-Nr. 2013-0114] and was conducted in accordance with the ethical standards laid down in the Declaration of Helsinki for human experimentation. The trial was registered at clinicaltrials.gov as NCT 01905345 (date of registration: 23/07/2013). Participants were recruited between $01 / 08 / 2013$ and $31 / 12 / 2015$ and the trial ended at $01 / 08 / 2017$ because of low accrual rate.

Study design. Participants were randomly assigned to one of two groups by drawing lots: 12 weeks of a habitual course observation phase followed by 12 weeks of progressive RT ( $\mathrm{HC} \rightarrow \mathrm{RT}, n=8)$ or 12 weeks of HIIT followed by 12 weeks of progressive RT (HIIT $\rightarrow$ RT, $n=9$ ). Participants were instructed to maintain their individual physical activity level and were advised not to include other types of systematic training or additional physical activities, which they were not already practicing before their enrolment. Before, between, and after the two 12 -week phases of the study, a percutaneous muscle biopsy was obtained from the $m$. vastus lateralis.

Training regimen. Participants performed three training sessions per week at the Institute of Human Movement Sciences, Exercise Physiology, University of Zurich Irchel. Training adherence was 85\% on average. All training sessions were supervised by a movement scientist. During the training sessions, participants were verbally encouraged by the supervisor. After each HIIT and RT session, participants received a drink containing $30 \mathrm{~g}$ of whey protein (Scientifics, Schwyz, Switzerland) in order to reduce variation in the intake of post-exercise protein and thereby the immediate anabolic response to the exercise training.

High-intensity interval training (HIIT). A HIIT session started with a 3-min warm-up at 50\% peak power (of a previously conducted incremental cycling test) on a bicycle ergometer (Bike XT, Technogym, Gambettola, Italy). Subsequently, power was increased to $65 \%$ peak power for $4 \mathrm{~min}$. Afterwards, participants conducted seven 1 -min high-intensity intervals at $85 \%$ peak power. Between the high-intensity intervals, power was reduced to $65 \%$ peak power for $4 \mathrm{~min}$ each time. As soon as a participant successfully completed the whole training session at the target values, power was increased by $3 \%$ peak power in the subsequent training session. The applied protocol was adopted from Charifi et al. ${ }^{16}$, using minor modifications to make the protocol more strenuous: $65 \%$ peak power instead of $65-75 \% \dot{V} O_{2 p e a k}$ and $85 \%$ peak power instead of $85-95 \% \dot{V} O_{2 p e a k}$.

Resistance training (RT). During RT, three exercises (leg extension, leg press, and squat) were conducted under supervision, assuring anatomically correct techniques. The participants performed three sets of each exercise with a 3-min resting period between sets. Every set was performed to volitional muscular failure. One repetition consisted of a 4-s shortening action, a 2-s isometric transition phase and a 4-s lengthening action. Each repetition had to be performed over the individual's full range of motion. In the first training session, participants started with approximately $60 \%$ of their previously determined 1-repetition maximum. In subsequent 
training sessions, the load was increased by the smallest possible unit, whenever participants exceeded a time under tension (TUT) of $120 \mathrm{~s}$ per set. For the leg extension exercise, an average TUT per set of $103 \pm 8 \mathrm{~s}$ for the $\mathrm{HC} \rightarrow \mathrm{RT}$ and $106 \pm 10 \mathrm{~s}$ for the HIIT $\rightarrow \mathrm{RT}$ group was accomplished. For the leg press exercise, a mean TUT per set of $112 \pm 15 \mathrm{~s}$ and $113 \pm 10 \mathrm{~s}$ was achieved for the $\mathrm{HC} \rightarrow \mathrm{RT}$ and HIIT $\rightarrow \mathrm{RT}$ group, respectively. The squat exercise resulted in a TUT per set of $103 \pm 12 \mathrm{~s}$ and $102 \pm 11 \mathrm{~s}$ for the $\mathrm{HC} \rightarrow \mathrm{RT}$ and HIIT $\rightarrow$ RT group, respectively. Between RT sessions, participants rested for at least $48 \mathrm{~h}$.

Muscle biopsy sampling. Muscle biopsies were taken at the University Hospital Zurich, Division of Neurology by an experienced physician. All biopsies were obtained from the $m$. vastus lateralis of the dominant leg after local anesthesia with $1 \%$ lidocaine, using a 6-mm Bergström needle (Dixons Surgical Instruments, Essex, UK) with suction applied. Biopsies served for the quantification of skeletal muscle capillarization and oxidative enzyme activity. All participants were instructed to refrain from exercise for $24 \mathrm{~h}$ before and $72 \mathrm{~h}$ after each biopsy. Initial biopsies were obtained from the mid-portion of the $m$. vastus lateralis, $\sim 18 \mathrm{~cm}$ proximal to the patella, approximating the midline of the quadriceps muscle group. The two subsequent biopsy procedures were performed at a distance of $1-2 \mathrm{~cm}$ proximal or distal to the site of the first biopsy in order to minimize effects of previous incisions. Muscle samples were immediately mounted in an embedding medium (Tissue-Tek, Sakura, Zoeterwoude, The Netherlands), snap frozen in nitrogen-cooled isopentane and subsequently stored at $-80^{\circ} \mathrm{C}$ until use.

Histochemistry. The $12 \mu \mathrm{m}$ cryocut cross-sections were enzyme-immunohistochemically stained using the monoclonal mouse anti-human CD31 endothelial antibody (DAKO, Carpinteria, CA, USA, 1:600) as marker for muscle capillaries, a VECTASTAIN ${ }^{\circledR}$ Elite ${ }^{\circledR}$ ABC-Peroxidase Kit (Mouse IgG; Vector laboratories, Burlingame, CA, USA) and Liquid DAB + Substrate Chromogen as peroxidase substrate (DAKO, Carpinteria, CA, USA, 1:50). Afterwards, sections were rinsed in tap water, counterstained in $0.1 \%$ Eosin Y (Sigma-Aldrich, Saint Louis, MO, USA), dried and mounted with Thermo Scientific ${ }^{\mathrm{TM}}$ Immu-Mount $^{\mathrm{TM}}$ (Thermo Fisher Scientific, Pittsburgh, PA, USA). For estimating muscle fiber oxidative capacity, sections were stained for succinate dehydrogenase (SDH) activity in sarcosomes. Thawed sections were incubated in phosphate buffered media $(\mathrm{pH} 7.6)$ containing the $\mathrm{SDH}$ substrate succinate in the form of succinic acid (Fluka Chemie, Buchs, Switzerland) and nitro blue tetrazolium chloride (Merck Millipore, Darmstadt, Germany) at $37^{\circ} \mathrm{C}$. Upon incubation, succinate is oxidized to fumarate by SDH revealing free hydrogen atoms, which in turn are used to reduce nitro blue tetrazolium to nitro blue diformazan. Nitro blue diformazan is insoluble in aqueous solutions and precipitates to purple aggregates at the place where it is formed. After incubation in media for $1.5-5 \mathrm{~h}$ at $37^{\circ} \mathrm{C}$ until desired stain intensity developed, sections were washed in PBS for $5 \mathrm{~min}$, dried and mounted with Thermo Scientific ${ }^{\mathrm{TM}} \mathrm{Immu}$-Mount ${ }^{\mathrm{TM}}$ (Thermo Fisher Scientific, Pittsburgh, PA, USA).

Muscle biopsy analyses. All muscle biopsy analyses were performed by a blinded investigator. The following indices were determined (Fig. 1): the number of capillaries surrounding a fiber (capillary contacts), the number of fibers sharing each capillary (sharing factor), and the individual capillary-to-fiber ratio, defined as the number of capillaries divided by their sharing factor, i.e. the number of full capillaries per individual fiber ${ }^{6}$. To examine the potential for blood-tissue exchange, the capillary density and the capillary-to-fiber perimeter exchange inde ${ }^{17}$ were calculated. Fiber CSA and perimeter were assessed by automatic calculation of the software after fully encircling the borders of individual muscle fibers. Fiber circularity was calculated using the formula $(4 \pi \cdot \mathrm{CSA}) /(\text { perimeter })^{2}$, and only fibers with a circularity higher than 0.65 were considered for analysis (perfect circle $=1.0$ ). On average, $68 \pm 15$ (week 0 ), $75 \pm 20$ (week 12), and $74 \pm 18$ (week 24) fibers were analyzed per biopsy sample for the overall capillary-to-fiber ratio. For all further capillarization indices, CSA, and perimeter, $62 \pm 12$ (week 0), $66 \pm 17$ (week 12), and $67 \pm 11$ (week 24) fibers were analyzed per biopsy sample. The staining protocol for SDH activity resulted in muscle fibers rich in SDH staining dark blue and densely granulated, and muscle fibers poor in SDH staining light blue and sparsely granulated. Quantification was performed by classifying fibers of a given section according to their relative staining intensity as fibers showing high (darkest staining), weak (intermediate staining) or no (weakest/no staining) SDH activity. All fibers on a given subset image showing distinct staining and limited signs of damage were considered for quantification resulting in $291 \pm 144$ (week 0), $260 \pm 78$ (week 12), and $335 \pm 130$ (week 24) fibers that were considered for analysis.

Sample size and statistical analyses. The necessary sample size was estimated to be 12 participants per group at a level of significance of $5 \%$ and a statistical power of $80 \%$. However, only a total of 20 participants could be recruited of which two withdrew from the study for personal reasons and one had to be excluded because he did not meet the training frequency guidelines. Data are presented as mean values \pm standard deviations (SD). Normality of data was ascertained by a Shapiro-Wilk test. Baseline values of the two groups were compared with an independent sample $t$-test. A paired sample $t$-test was performed to detect changes during the habitual course observation phase. A univariate general linear model was applied to examine the changes that occurred during the RT and HIIT phase. For this analysis, the differences post-pre $(\Delta)$ of each variable was compared between groups. Significant differences within groups from pre- to post-phase were assessed by parameter estimates. The effect of the preceding habitual course observation phase or HIIT on RT adaptation was analyzed with ANCOVA. We analyzed the differences between the groups by using the $\Delta$ parameter of phase 1 (habitual course observation phase or HIIT) as a covariate to account for the alterations that occured during phase 1 . The level of significance was set at $P<0.05$. The effect size was described as $\eta_{\mathrm{p}}^{2}$ (partial eta-squared). All statistical analyses were performed using the software SPSS Statistics 24.0 (SPSS, Chicago, IL, USA). 

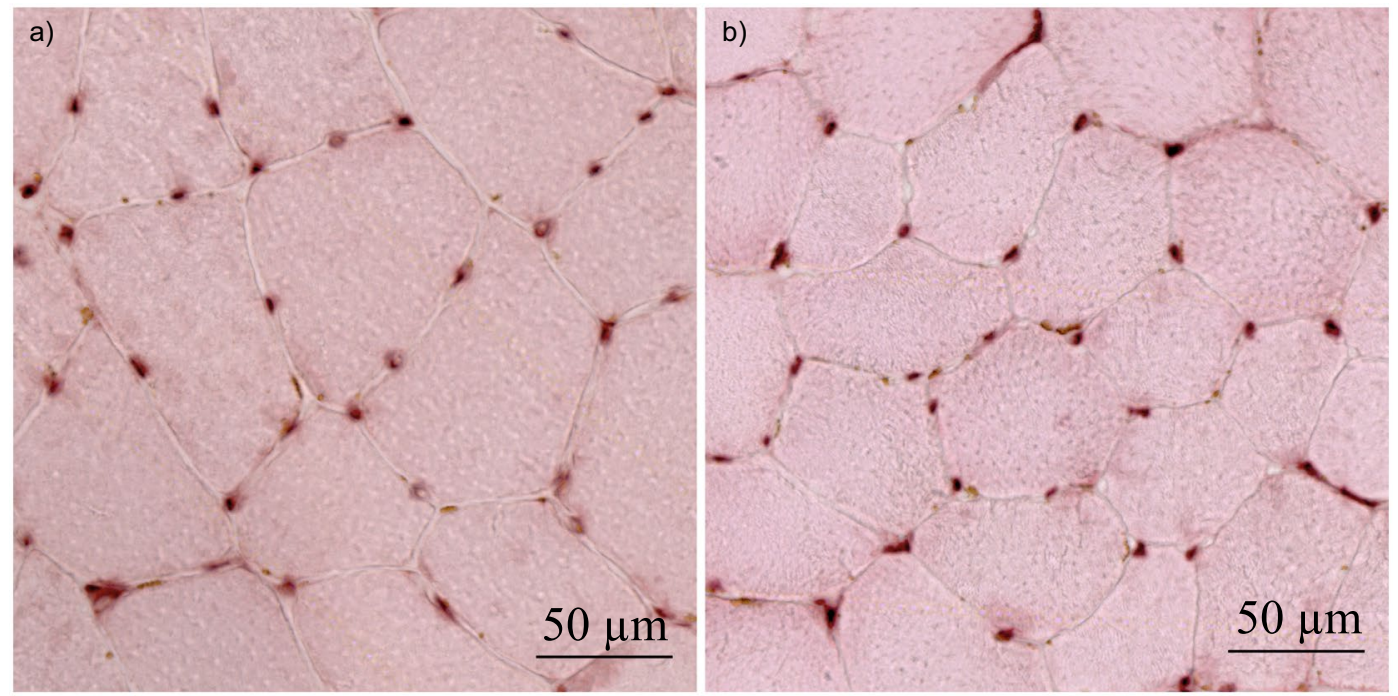

c)

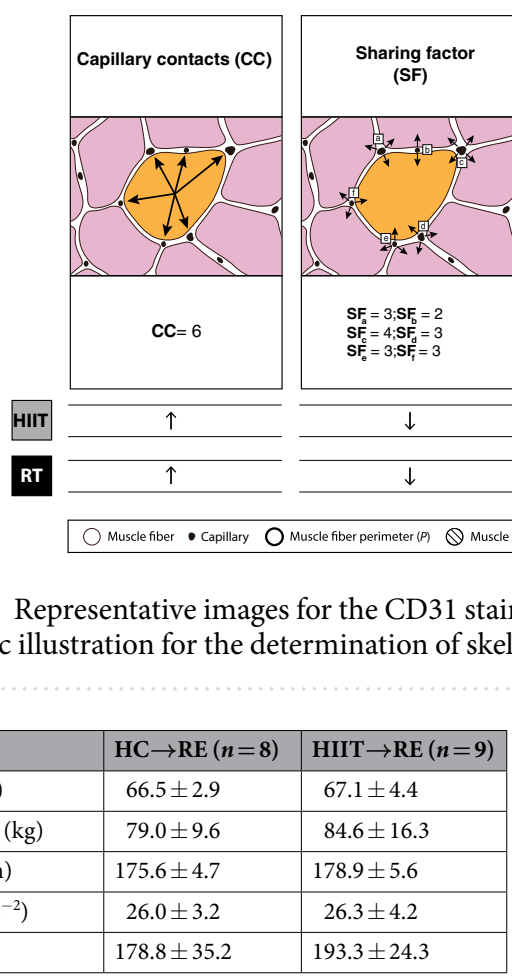

Table 1. Participants' characteristics and functional measures at baseline for two groups with different exercise intervention protocols. Data are mean \pm SD. Abbreviations: BMI, body mass index; $\dot{V} O_{2 \text { peak }}$, peak oxygen uptake; $P_{\text {peak }}$, peak power.

Compliance with ethical standards. Ethical approval. All procedures in the study involving human participants were in accordance with the ethical standards of the institutional research committee and with the 1964 Helsinki declaration and its later amendments.

Informed consent. Informed consent was obtained from all individual participants included in the study.

\section{Results}

Participant's characteristics and habitual course observation phase. Participants of the two groups did not differ in physical characteristics and functional measures at baseline (Table 1). During the habitual course observation phase, all capillarization indices remained constant (Table 2). Furthermore, there was no alteration in overall CSA and overall muscle fiber perimeter (Table 2). The proportion of muscle fibers displaying high, weak, and no SDH activity also remained unaltered (Table 3 ). 


\begin{tabular}{|l|c|l|}
\hline & week 0 & week 12 \\
\hline Overall capillary-to-fiber ratio & $1.77 \pm 0.30$ & $1.78 \pm 0.34$ \\
\hline Capillary contacts & $5.12 \pm 0.70$ & $5.25 \pm 0.80$ \\
\hline Sharing factor & $2.75 \pm 0.11$ & $2.75 \pm 0.10$ \\
\hline Individual capillary-to-fiber ratio & $1.91 \pm 0.34$ & $1.94 \pm 0.35$ \\
\hline Capillary density $\left(\right.$ capillaries $\left.\cdot \mathrm{mm}^{-2}\right)$ & $384 \pm 40$ & $401 \pm 47$ \\
\hline Capillary-to-fiber perimeter exchange index (capillaries-1000 $\left.\mu^{-1}\right)$ & $6.44 \pm 0.73$ & $6.59 \pm 0.85$ \\
\hline Cross-sectional area $\left(\mu \mathrm{m}^{2}\right)$ & $5117 \pm 853$ & $5024 \pm 711$ \\
\hline Perimeter $(\mu \mathrm{m})$ & $295 \pm 26$ & $294 \pm 19$ \\
\hline
\end{tabular}

Table 2. Results of the habitual course observation phase. Data are mean $\pm \operatorname{SD}$ for $n=8$ older men.

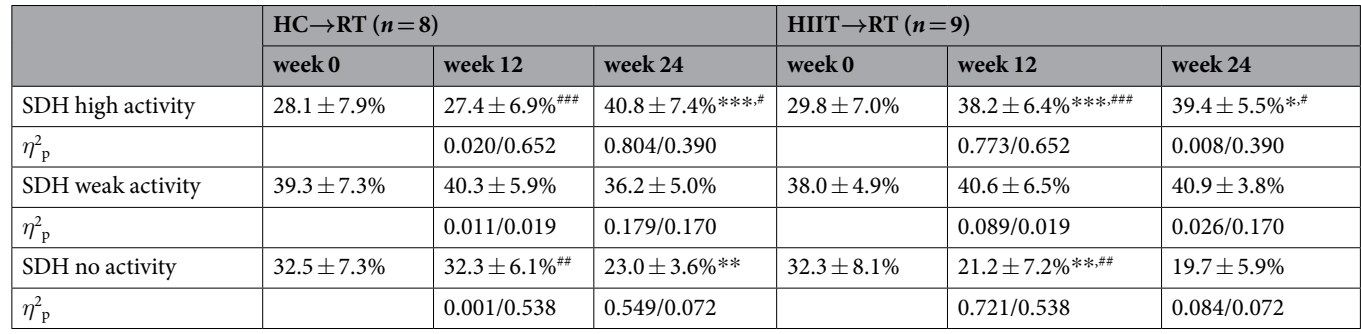

Table 3. Succinate dehydrogenase $(\mathrm{SDH})$ activity in both training groups at the three points in time. Data are mean $\pm \mathrm{SD}$. HC $\rightarrow \mathrm{RT}$, habitual course observation phase (HC, 12 weeks) followed by resistance training (RT, 12 weeks); HIIT $\rightarrow$ RT, consecutive training regimens of high-intensity interval training (HIIT, 12 weeks) followed by RT ( 12 weeks). $* P<0.05, * * P<0.01, * * * P<0.001$, significant differences versus previous point in time within group; ${ }^{\#} P<0.05,{ }^{\# \#} P<0.01$, ${ }^{\# \#} P<0.001$, significant group $\mathrm{x}$ time interaction for previous phase. $\eta_{\mathrm{p}}^{2}$ is displayed for within group comparisons before slash and for group $\mathrm{x}$ time interactions after slash.

\begin{tabular}{|c|c|c|c|c|c|c|}
\hline & \multicolumn{3}{|l|}{ RT $(n=8)$} & \multicolumn{3}{|c|}{ HIIT $(n=9)$} \\
\hline & $\begin{array}{l}\text { pre- } \\
\text { training }\end{array}$ & post-training & $\eta_{\mathrm{p}}^{2}$ & $\begin{array}{l}\text { pre- } \\
\text { training }\end{array}$ & post-training & $\eta_{\mathrm{p}}^{2}$ \\
\hline Overall capillary-to-fiber ratio & $1.78 \pm 0.34$ & $1.96 \pm 0.26 * *$ & 0.428 & $1.59 \pm 0.30$ & $1.82 \pm 0.20^{* * *}$ & 0.577 \\
\hline Capillary contacts & $5.25 \pm 0.80$ & $5.79 \pm 0.63^{* *}$ & 0.452 & $4.70 \pm 0.84$ & $5.33 \pm 0.49^{* *}$ & 0.562 \\
\hline Sharing factor & $2.75 \pm 0.10$ & $2.71 \pm 0.08^{*}$ & 0.262 & $2.80 \pm 0.11$ & $2.73 \pm 0.09 * *$ & 0.448 \\
\hline Individual capillary-to-fiber ratio & $1.94 \pm 0.35$ & $2.15 \pm 0.30 * *$ & 0.414 & $1.73 \pm 0.36$ & $1.97 \pm 0.22 * *$ & 0.541 \\
\hline Capillary density (capillaries $\cdot \mathrm{mm}^{-2}$ ) & $401 \pm 47$ & $374 \pm 32$ & 0.139 & $360 \pm 43$ & $365 \pm 40$ & 0.005 \\
\hline $\begin{array}{l}\text { Capillary-to-fiber perimeter exchange index } \\
\left(\text { capillaries } 1000 \mu \mathrm{m}^{-1}\right)\end{array}$ & $6.59 \pm 0.85$ & $6.72 \pm 0.55$ & 0.034 & $5.84 \pm 0.49$ & $6.35 \pm 0.53 * *$ & 0.385 \\
\hline
\end{tabular}

Table 4. Capillarization indices before and after 12 weeks of resistance training (RT) or high-intensity interval training (HIIT). Data are mean \pm SD. $* P<0.05$, $* * P<0.01$, $* * * P<0.001$, significantly different to pre-training within group. $\eta_{\mathrm{p}}^{2}$ is displayed for the within group differences from pre- to post-training.

Differences between RT and HIIT. RT and HIIT resulted in significant increases in most capillarization indices compared to pre-training values (Table 4). There were no significant differences between single periods of RT and HIIT in the increases in overall capillary-to-fiber ratio $\left(P=0.517, \eta_{\mathrm{p}}^{2}=0.029\right)$ and capillary contacts $\left(P=0.659, \eta_{\mathrm{p}}^{2}=0.013\right)$, in the decrease in the sharing factor $\left(P=0.510, \eta_{\mathrm{p}}^{2}=0.029\right)$, and in the increase in the individual capillary-to-fiber ratio $\left(P=0.614, \eta_{\mathrm{p}}^{2}=0.017\right)$. Capillary density remained constant during both training interventions, without a difference between RT and HIIT $\left(P=0.208, \eta_{\mathrm{p}}^{2}=0.103\right)$. Capillary-to-fiber perimeter exchange index was solely increased through HIIT, but there was no significant group by time interaction $\left(P=0.136, \eta_{\mathrm{p}}^{2}=0.142\right)$. Overall CSA was significantly increased by RT (pre-training: $5024 \pm 711$ $\mu \mathrm{m}^{2}$, post-training: $5911 \pm 839 \mu \mathrm{m}^{2}, P=0.040, \eta_{\mathrm{p}}^{2}=0.251$ ), while it was not significantly altered during HIIT (pre-training: $5052 \pm 1576 \mu \mathrm{m}^{2}$, post-training: $5666 \pm 1022 \mu \mathrm{m}^{2}, P=0.120, \eta_{\mathrm{p}}^{2}=0.153$ ). The proportion of muscle fibers with high SDH activity increased significantly by RT and HIIT (Table 3), whereas the proportion of muscle fibers with no SDH activity was significantly reduced by both training modalities. There were no alterations in the proportion of muscle fibers with weak SDH activity during the whole intervention phase (Table 3).

Differences between RT without prior HIIT or subsequent to HIIT. Most capillarization indices (Fig. 2a-e), CSA (Fig. 2g), and fiber perimeter (Fig. 2h) remained constant during RT with prior HIIT. Capillary-to-fiber perimeter exchange index increased significantly during RT with prior HIIT (Fig. 2f). There were no significant group $\mathrm{x}$ time interactions between RT performed with or without prior HIIT for 
overall capillary-to-fiber ratio $\left(P=0.618 ; \eta_{\mathrm{p}}^{2}=0.022\right)$, capillary contacts $\left(P=0.350 ; \eta_{\mathrm{p}}^{2}=0.084\right)$, sharing factor $\left(P=0.322 ; \eta_{\mathrm{p}}^{2}=0.051\right)$, individual capillary-to-fiber ratio $\left(P=0.521 ; \eta_{\mathrm{p}}^{2}=0.042\right)$, capillary density $(P=0.306$; $\left.\eta_{\mathrm{p}}^{2}=0.061\right)$, capillary-to-fiber perimeter exchange index $\left(P=0.314 ; \eta_{\mathrm{p}}^{2}=0.051\right)$, CSA $\left(P=0.114 ; \eta_{\mathrm{p}}^{2}=0.174\right)$, and fiber perimeter $\left(P=0.150 ; \eta_{\mathrm{p}}^{2}=0.141\right)$. The proportion of muscle fibers displaying high $(P=0.741$; $\left.\eta_{\mathrm{p}}^{2}=0.008\right)$, weak $\left(P=0.554 ; \eta_{\mathrm{p}}^{2}=0.026\right)$, and no SDH activity $\left(P=0.277 ; \eta_{\mathrm{p}}^{2}=0.084\right)$ remained constant during RT with prior HIIT.

Harms or unintended effects. None of the participants did experience harms or unintended effects during the study, neither due to the training interventions nor due to the muscle biopsy sampling.

\section{Discussion}

In the present study, we found that single periods of exclusive RT or HIIT caused a similar increase of determinants for capillarization and SDH activity in older men. In addition, improved capillarization indices and SDH activity after 12 weeks of HIIT were maintained during subsequent 12 weeks of RT.

RT and HIIT without prior training resulted in similar increases in overall capillary-to-fiber ratio, capillary contacts, and individual capillary-to-fiber ratio. These results suggest that both training modalities were effective in inducing angiogenesis. The increase in capillaries per fiber was associated with a similar decrease in the sharing factor in both training groups. Before the training interventions, the majority of capillaries was positioned at the junction of three individual muscle fibers. During the training intervention, novel capillaries were primarily built alongside the border of only two muscle fibers. Hence, new capillaries were mainly formed at positions at which they shared a minimum of muscle fibers. This leads to an optimized exchange surface and a higher capacity for blood-tissue exchange. However, capillary density was not significantly altered following the intervention period of both training modalities. The significant increase of CSA in the RT group and the slight non-significant increase of CSA in the HIIT group might explain the constant capillary densities. In contrast, capillary-to-fiber perimeter exchange index was increased during HIIT. This was not the case following RT, as the significant increase in overall muscle fiber perimeter by RT prevented a significant increase in capillary-to-fiber perimeter exchange index. Important to note, the HIIT $\rightarrow$ RT group had a markedly lower baseline value for the capillary-to-fiber perimeter exchange index compared to the $\mathrm{HC} \rightarrow \mathrm{RT}$ group, and even the significant increase in the capillary-to-fiber perimeter exchange index during HIIT was not sufficient to reach the values of the $\mathrm{HC} \rightarrow \mathrm{RT}$ group. Moreover, during the second phase of the study, the RT period following the HIIT period, resulted again in a significant increase in the capillary-to-fiber perimeter exchange index, indicating that RT can in fact elevate this index. Thus, the observed difference in the adaptation of the capillary-to-fiber perimeter exchange index between HIIT and RT without prior training seems to be explained by uneven, though not significant, baseline values, and not necessarily because there is an actual difference in the underlying adaptation mechanisms between HIIT and RT. The changes in SDH activity observed during HIIT and RT without prior training further support the notion that the two training modalities share similarities in their mechanisms of adaptation. Following both types of training, the percentage of muscle fibers with high SDH activity was increased, indicating that oxidative energy metabolism was improved with both HIIT and RT. Hence, differences in oxygen flux in response to the distinct training modalities, as well as a specific adaptation of the capillary-to-fiber perimeter exchange index to HIIT seems unlikely.

Additional supportive evidence for similar adaptations in HIIT and RT arises from the results of the sequential training design. The increased capillarization indices and the improvements in oxidative enzyme activity achieved with HIIT were both preserved during 12 weeks of subsequent RT. Moreover, the capillary-to-fiber perimeter exchange index was even further increased during subsequent RT, and relative values for all other variables pointed in the same direction as in the previous HIIT phase. While it is known that capillary density can be maintained during short periods of detraining ${ }^{18}$, oxidative enzyme activity returns to baseline levels after only 2 weeks of detraining following an 8 -week training program ${ }^{19}$. Specifically, oxidative enzyme activity follows an initial large decrease after training cessation and stabilizes thereafter at a level higher than prior to training ${ }^{20}$. Thus, our results demonstrate that RT is a sufficient stimulus to maintain or even improve HIIT-induced adaptations in capillarization indices and oxidative enzyme activity. However, because of the impossibility to allocate a specific identity to individual capillaries with the techniques applicable to human biopsies, it is not possible to determine whether the capillaries counted after RT are the same as built during prior HIIT, i.e. whether single capillaries or the total number could in fact be maintained. Hence, if the mechanisms underlying capillary formation are different between HIIT and RT, it is conceivable that the capillaries formed during HIIT were deconditioned after HIIT cessation and replaced by de novo formation via different mechanisms induced by the RT stimulus. Despite these potential limitations for interpretation, our data does not suggest that the two training modalities have strong additive effects on vascularization. This is in accordance with the only other study that used a sequential training design, wherein the reverse order of the same two training modalities, i.e. RT $\rightarrow \mathrm{ET}$, was investigated $^{6}$. In that study, individual capillary-to-fiber ratio and capillary contacts increased during RT and were not further improved by a subsequent period of ET. Interestingly, however, capillary-to-fiber perimeter exchange index increased during RT and was further elevated during the following period of ET ${ }^{6}$. The further increase in capillary-to-fiber perimeter exchange index during ET may be explained by the significant decrease in muscle fiber perimeter that occurred coincidently ${ }^{6}$.

The increased capillarization after both, 12 weeks of HIIT or 12 weeks of RT has at least three functional benefits for older men. First, enhanced capillarization allows for an improved blood-tissue exchange and may result in greater glucose delivery and metabolization in skeletal muscle. This is consistent with previous observations revealing a favorable association between muscle capillarization and insulin sensitivity in older adults ${ }^{18,21-23}$. Moreover, the increase in capillary density has previously been shown to significantly correlate with higher insulin sensitivity in older adults following 6 months of $\mathrm{ET}^{17}$. Second, enhanced capillarization might increase leg 
a)

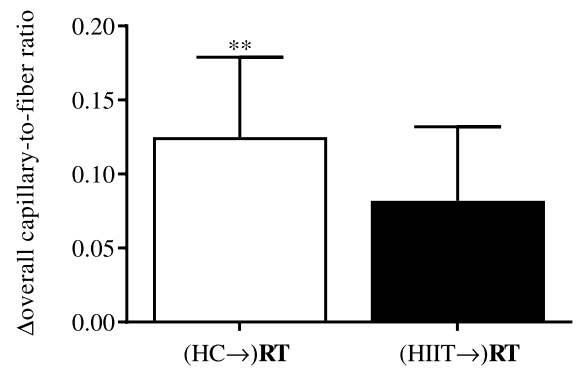

c)

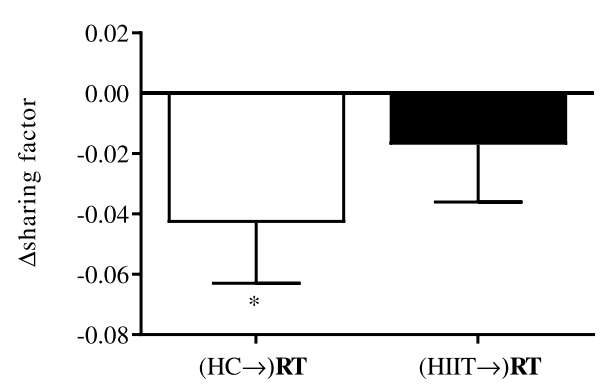

e)

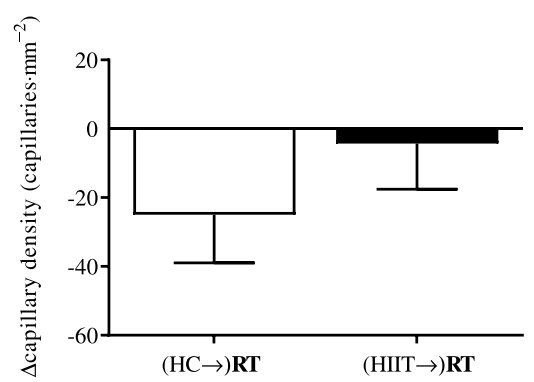

g)

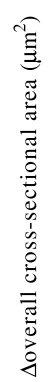

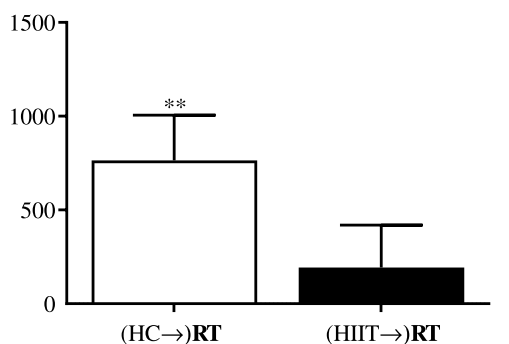

b)

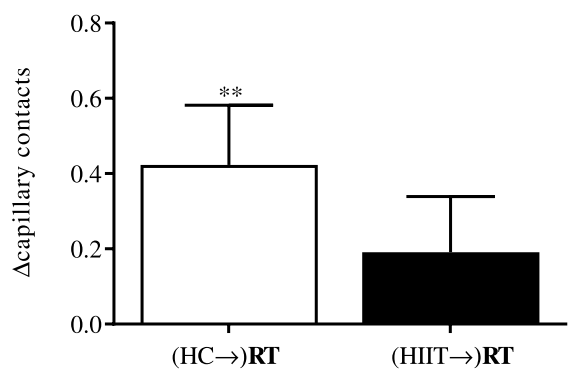

d)
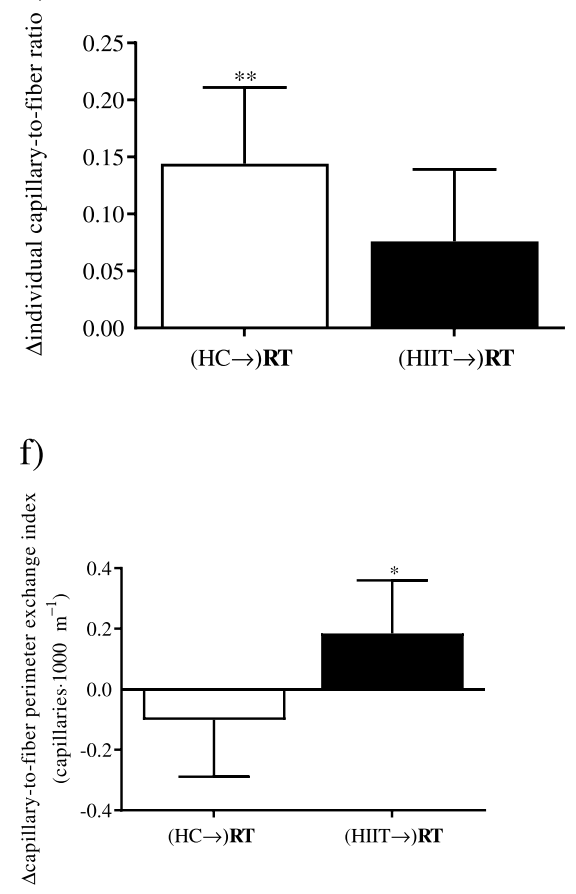

h)

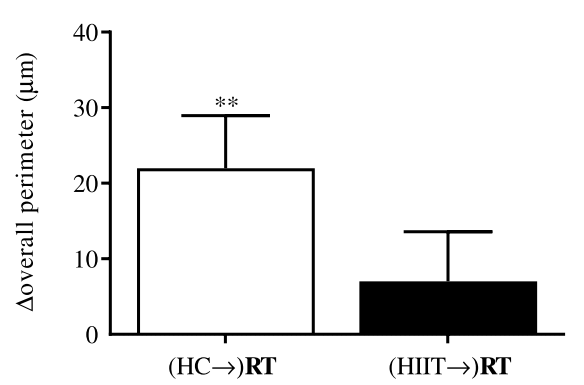

Figure 2. Resistance training-induced changes in the $\mathrm{HC} \rightarrow \mathrm{RT}$ (white bars) and HIIT $\rightarrow \mathrm{RT}$ (black bars) groups for (a) overall capillary-to-fiber ratio, (b) capillary contacts, (c) sharing factor, (d) individual capillary-to-fiber ratio, (e) capillary density, (f) capillary-to-fiber perimeter exchange index, (g) overall cross-sectional area, (h) overall perimeter. Bars and error bars represent mean and standard deviations. $* P<0.05, * * P<0.01$, significant change within group in the training period.

blood flow in response to exercise, which is supported by a previous study showing increased leg blood flow and leg vascular conductance in response to exercise and feeding after 20 weeks of RT in older adults ${ }^{24}$. An increased femoral artery blood flow has the potential to attenuate muscle protein breakdown following exercise and feeding and might turn net protein balance into positive numbers ${ }^{25}$. Furthermore, the number of muscle fiber capillary contacts has been shown to correlate with myofibrillar protein synthetic response to a mixed meal ${ }^{26}$. Third, it has 
been proposed that blood supply is a critical factor for the maintenance or gain of muscle mass in young ${ }^{27}$ and older adults ${ }^{8,9}$ in response to RT. Taken together, based on the beneficial effects of improved capillarization listed above, and in conjunction with our own findings, both HIIT and RT appear to be effective countermeasures to mitigate age-related metabolic impairments and decreases in skeletal muscle mass with increasing age.

Our findings are supported by previously published data, showing that constant moderate intensity ET is an adequate stimulus to substantially induce angiogenic adaptations in older adults ${ }^{6,7,11}$. We extend the scientific knowledge, showing that a HIIT protocol improved capillarization indices in older men. Furthermore, we observed a significant decrease in the sharing factor, which has previously been reported to be non-significantly lowered following constant moderate intensity ET ${ }^{6,7}$. Contrary to the uniform findings on the effects of ET on capillarization, previous studies on the effects of RT on capillarization indices were less consistent. Our results are in line with a few studies that reported an increase in capillarization indices after 9-12 weeks of $\mathrm{RT}^{6,9,12}$, but are in contrast to other studies that showed no significant increases after 12-24 weeks of $\mathrm{RT}^{8,13}$. Unfortunately, it is not possible to identify the reasons for this discrepancy, because the RT protocols of these studies do not provide sufficient details for comparisons. While information about exercise types, sets, repetitions, and training load is provided, details on other mechano-biological descriptors of resistance exercise stimuli ${ }^{28}$ are lacking. These descriptors, including the fractional and temporal distribution of muscle actions per repetition, the duration of one repetition, and the time under tension (TUT), are substantially determining the character of the applied stimulus, which in turn affects the molecular patterns induced in exercised muscle fibers. In particular, TUT (of one single set and/or summed up for a given muscle over a complete training session) may play a pivotal role in stimulating capillarization with RT, and could have decisively varied between studies. Performing a RT exercise with a prolonged TUT and as close as possible to volitional muscular failure (as it was the case with our RT protocol), may increase hypoxic, mechanical and metabolic stress, and thereby stimulate angiogenesis in a sustained manner. In contrast, when TUT is too short and/or the exercise is not performed to volitional muscular failure, the stresses might be lower and thus the angiogenic stimulus weaker. Moreover, prolonged TUT may be necessary to recruit and fatigue high threshold motor units, and thereby to stimulate angiogenesis in fast (i.e. MyHC-2) muscle fibers more effectively. Nevertheless, to draw firm conclusions regarding the role of TUT or other mechano-biological descriptors, further studies are required, which vary these factors and directly compare their effects on capillarization.

Limitations of the study. We have chosen a parallel group study design, since this represents the most feasible setting with human participants. The most accurate study design to compare the training effects of both training modalities and their sequential application is a randomized crossover design. However, according to current knowledge on the persistence of muscular adaptations in conjunction with skeletal muscle memory, the use of this study design is not appropriate for human participants. While muscle memory in response to ET was questioned recently ${ }^{29}$, facilitated mitochondrial remodelling was observed after $\mathrm{RT}^{30}$ and training-induced increases in the number of myonuclei are long-lasting, resulting in an altered precondition for future investigations $^{31}$. In previous studies, it has been proposed that the lifespan of human myonuclei is at least 15 years $^{31,32}$. Consequently, the notion of a washout period between the two arms of the crossover design and its application in human participants is at least questionable.

A further limitation of the current study is that only male participants have been investigated. Recent research has suggested that sex may affect the acute exercise response and/or long-term exercise adaptation including the vascular system ${ }^{33,34}$. Therefore, our results do not necessarily apply for both sexes of the elderly population and more studies are required to clarify this.

\section{Conclusion}

A 12-week period of exclusive RT without prior training resulted in similar improvements in most capillarization indices and SDH activity as compared to 12 weeks of exclusive HIIT. Furthermore, the HIIT-induced improvements in capillarization and oxidative enzyme activity were maintained during a subsequent 12 -week RT intervention. Hence, the applied RT protocol represents a sufficient stimulus to preserve the HIIT-induced increase in capillarization indices. Collectively, our study provides evidence that both RT and HIT can be valid training modalities to induce angiogenesis and improve muscle fiber capillarization indices in older men. These adaptations may help to prevent the development of sarcopenia and metabolic impairments with increasing age.

Received: 20 January 2020; Accepted: 1 April 2020;

Published online: 20 April 2020

\section{References}

1. Landers-Ramos, R. Q. \& Prior, S. J. The microvasculature and skeletal muscle health in aging. Exerc. Sport. Sci. Rev. 46, 172-179 (2018).

2. Wilson, T. M. \& Tanaka, H. Meta-analysis of the age-associated decline in maximal aerobic capacity in men: relation to training status. Am. J. Physiol. Heart. Circ. Physiol. 278, H829-H834 (2000).

3. Kumar, V. et al. Age-related differences in the dose-response relationship of muscle protein synthesis to resistance exercise in young and old men. J. Physiol. 587, 211-217 (2009).

4. Moore, D. R. et al. Protein ingestion to stimulate myofibrillar protein synthesis requires greater relative protein intakes in healthy older versus younger men. J. Gerontol. A. Biol. Sci. Med. Sci. 70, 57-62 (2015).

5. Shad, B. J., Thompson, J. L. \& Breen, L. Does the muscle protein synthetic response to exercise and amino acid-based nutrition diminish with advancing age? A systematic review. Am. J. Physiol. Endocrinol. Metab. 311, E803-817 (2016).

6. Hepple, R. T., MacKinnon, S. L. M., Goodman, J. M., Thomas, S. G. \& Plyley, M. J. Resistance and aerobic training in older men: effects on $V \mathrm{O}_{2 \text { peak }}$ and the capillary supply to skeletal muscle. J. Appl. Physiol. 82, 1305-1310 (1997).

7. Gavin, T. P. et al. No difference in the skeletal muscle angiogenic response to aerobic exercise training between young and aged men. J. Physiol. 585, 231-239 (2007). 
8. Snijders, T. et al. Muscle fibre capillarization is a critical factor in muscle fibre hypertrophy during resistance exercise training in older men. J. Cachexia. Sarcopenia. Muscle. 8, 267-276 (2017).

9. Verdijk, L. B., Snijders, T., Holloway, T. M., Van Kranenburg, J. \& Van Loon, L. J. C. Resistance Training Increases Skeletal Muscle Capillarization in Healthy Older Men. Med. Sci. Sports. Exerc. 48, 2157-2164 (2016).

10. Prior, S. J. et al. Sarcopenia is associated with lower skeletal muscle capillarization and exercise capacity in older adults. J. Gerontol. A. Biol. Sci. Med. Sci. 71, 1096-1101 (2016).

11. Coggan, A. R. et al. Skeletal muscle adaptations to endurance training in 60- to 70-yr-old men and women. J. Appl. Physiol. 72, $1780-1786$ (1992).

12. Frontera, W. R., Meredith, C. N., O’Reilly, K. P. \& Evans, W. J. Strength training and determinants of $V \mathrm{O}_{2 \max }$ in older men. J. Appl. Physiol. 68, 329-333 (1990).

13. Hagerman, F. C. et al. Effects of high-intensity resistance training on untrained older men. I. Strength, cardiovascular, and metabolic responses. J. Gerontol. A. Biol. Sci. Med. Sci. 55, B336-346 (2000).

14. Weber, M. A. et al. Concentric resistance training increases muscle strength without affecting microcirculation. Eur. J. Radiol. 73, 614-621 (2010).

15. Gibala, M. J., Little, J. P., Macdonald, M. J. \& Hawley, J. A. Physiological adaptations to low-volume, high-intensity interval training in health and disease. J. Physiol. 590, 1077-1084 (2012).

16. Charifi, N., Kadi, F., Féasson, L. \& Denis, C. Effects of endurance training on satellite cell frequency in skeletal muscle of old men. Muscle. Nerve. 28, 87-92 (2003).

17. Hepple, R. T. A new measurement of tissue capillarity: the capillary-to-fibre perimeter exchange index. Can. J. Appl. Physiol. 22, 11-22 (1997).

18. Prior, S. J. et al. Increased skeletal muscle capillarization independently enhances insulin sensitivity in older adults after exercise training and detraining. Diabetes. 64, 3386-3395 (2015).

19. Henriksson, J. \& Reitman, J. S. Time course of changes in human skeletal muscle succinate dehydrogenase and cytochrome oxidase activities and maximal oxygen uptake with physical activity and inactivity. Acta. Physiol. Scand. 99, 91-97 (1977).

20. Neufer, P. D. The effect of detraining and reduced training on the physiological adaptations to aerobic endurance training. Sports. Med. 8, 302-321 (1989).

21. Hedman, A., Berglund, L., Essen-Gustavsson, B., Reneland, R. \& Lithell, H. Relationships between muscle morphology and insulin sensitivity are improved after adjustment for intra-individual variability in 70-year-old men. Acta. Physiol. Scand. 169, 125-132 (2000).

22. Snijders, T. et al. Muscle fiber capillarization as determining factor on indices of insulin sensitivity in humans. Phyisol. Rep. 5, e13278 (2017)

23. Solomon, T. P., Haus, J. M., Li, Y. \& Kirwan, J. P. Progressive hyperglycemia across the glucose tolerance continuum in older obese adults is related to skeletal muscle capillarization and nitric oxide bioavailabiltiy. J. Clin. Endocrinol. Metab. 96, 1377-1384 (2011).

24. Phillips, B. et al. Resistance exercise training improves age-related declines in leg vascular conductance and rejuvenates acute leg blood flow responses to feeding and exercise. J. Appl. Physiol. 112, 347-353 (2012).

25. Phillips, B. E. et al. The effects of resistance exercise training on macro- and micro-circulatory responses to feeding and skeletal muscle protein anabolism in older men. J. Physiol. 593, 2721-2734 (2015).

26. Moore, D. R. et al. Low-load reistance exercise during inactivity is associated with greater fibre area and satellite cell expression in older skeletal muscle. J. Cachexia. Sarcopenia. Muscle. 9, 747-754 (2018).

27. Holloway, T. M., Snijders, T., van Kranenburg, J., van Loon, J. C. \& Verdijk, L. B. Temporal response of angiogenesis and hypertrophy to resistance training in young men. Med. Sci. Sports. Exerc. 50, 36-45 (2018).

28. Toigo, M. \& Boutellier, U. New fundamental resistance exercise determinants of molecular and cellular adaptations. Eur. J. Appl. Physiol. 97, 643-663 (2006).

29. Lindholm, M. E. et al. The impact of endurance training on human skeletal muscle memory, global isoform expression and novel transcripts. PLOS. Genet. 12, e1006294 (2016).

30. Lee, H. et al. A cellular mechanism of muscle memory facilitates mitochondrial remodeling following resistance training. J. Physiol. 596, 4413-4426 (2018)

31. Gundersen, K. Muscle memory and a new cellular model for muscle atrophy and hypertrophy. J. Exp. Biol. 219, 235-242 (2016).

32. Spalding, K. L., Bhardwaj, R. D., Buchholz, B. A., Druid, H. \& Frisen, J. Retrospective birth dating of cells in humans. Cell. 122, 133-143 (1995)

33. Santos-Parker, J. R., Strahler, T. R., Vorwald, V. M., Pierce, G. L. \& Seals, D. R. Habitual aerobic exercise does not protect against micro- or macrovascular endothelial dysfunction in healthy estrogen-deficient postmenopausal women. J. Appl. Physiol. 122, 11-19 (2017).

34. Yoo, J. K. et al. Sex impacts the flow-mediated dilation response to acute aerobic exercise in older adults. Exp. Gerontol. 91, 57-63 (2017).

\section{Acknowledgements}

We thank the participants for their effort. We also thank D. Paganini and C. Viecelli for their assistance during the training intervention. Imaging was performed with equipment maintained by the Center for Microscopy and Image Analysis, University of Zurich. Institutional affiliations paid the researchers' salaries, infrastructure and consumables costs. Study participants were not remunerated for their participation.

\section{Author contributions}

A.B.L., S.M.M., D.A. and M.T. conceived and designed research. A.B.L., S.M.M., D.A., J.A.P., M.L.A. and H.H.J. were involved in data collection and/or conducted experiments. A.B.L., S.M.M., M.F. and M.T. analyzed and interpreted data. A.B.L., S.M.M., and M.T. wrote the manuscript. All authors critically reviewed the manuscript and approved the final version of the manuscript.

\section{Competing interests}

The authors declare no competing interests.

\section{Additional information}

Correspondence and requests for materials should be addressed to M.T.

Reprints and permissions information is available at www.nature.com/reprints. 
Publisher's note Springer Nature remains neutral with regard to jurisdictional claims in published maps and institutional affiliations.

(c) (i) Open Access This article is licensed under a Creative Commons Attribution 4.0 International License, which permits use, sharing, adaptation, distribution and reproduction in any medium or format, as long as you give appropriate credit to the original author(s) and the source, provide a link to the Creative Commons license, and indicate if changes were made. The images or other third party material in this article are included in the article's Creative Commons license, unless indicated otherwise in a credit line to the material. If material is not included in the article's Creative Commons license and your intended use is not permitted by statutory regulation or exceeds the permitted use, you will need to obtain permission directly from the copyright holder. To view a copy of this license, visit http://creativecommons.org/licenses/by/4.0/.

(c) The Author(s) 2020 